\title{
The Clinical Features of Spasms in Patients with a Cervical Cord Injury
}

\author{
J. Kawamura, $M D,{ }^{1}$ M. Ise, $M D,{ }^{2}$ and $M$. Tagami ${ }^{3}$ \\ ${ }^{1}$ Director in Department of Rehabilitation, Osaka Rosai Hospital, 1179-3, \\ Nagasone-cho, Sakai, Osaka, 591, Fapan, ${ }^{2}$ Instructor in Department of Rehabilita- \\ tion, Kawasaki Medical School, 557, Matsushima, Kurashiki-shi, Okayama-ken, \\ 701-01, Fapan ${ }^{3}$ Physical therapist in Department of Rehabilitation, Osaka Rosai \\ Hospital, $\mathcal{F a p a n}$.
}

\begin{abstract}
Summary
A study concerning subjective symptoms of spasms which occur during 24 hours was carried out in 13 quadriplegic patients. All had spasms. Tonic and clonic spasms occurred involving their limbs and trunk. Extensor spasms occurred more often in the lower limbs than in the upper limbs. The duration of spasms was 8 seconds on average and the frequency was 15 times on average per day. The spasms which were severe tended to last longer in duration and were more frequent in occurrence. No spasms occurred without a trigger. The activities in daily living were interfered with by spasms in most patients. Most of these activities also triggered spasms.
\end{abstract}

Key words: Spinal cord injuries; Spasms; Quadriplegia; Subjective symptoms.

The Osaka Rosai Hospital in Japan is one of 38 hospitals for patients injured by an industrial accident. Although there are 750 beds in all, about 50 patients with spinal cord injuries are usually admitted to the hospital, including some patients who remain even after completion of their medical rehabilitation. Some patients have spasms in their limbs and trunk as well as pain. The purpose of this paper is to highlight the features of subjective symptoms of spasms during 24 hours for patients with a cervical cord injury.

\section{Material and methods}

There were 13 quadriplegic patients at the Osaka Rosai Hospital at the time of this study. All were male between the ages 16 and 56 years (a median age of 44 years). They had a cervical cord injury 6 months to 23 years ( 5 years on average) prior to this study. Five of 13 patients were at C4 level, 2 at C5, 5 at C6, and 1 at C7. Nine of the 13 patients had a complete lesion, and 4 had an incomplete lesion. None could walk and all were dependent on attendants for all activities 
of daily living except for eating using self-help devices, and to move around with an ordinary or powered wheelchair. They were in excellent physical condition at the time of this study and none had severe complications, such as decubitus uclers or a urinary infection.

Subjective symptoms of spasms occurring during 24 hours were inquired about through individual interviews by the physical therapist who is one of the authors of this paper.

\section{Results}

Spasms were found in the upper limbs in 11 patients, and in the lower limbs in all, and in the trunk in all. Severe spasms were complained of by 7 patients, moderate by 3 patients, and slight by 3 patients. The duration of spasms felt by individual patients ranged from 3 to 30 seconds (average 8 seconds). The frequency of spasms during 24 hours ranged from 5 to 30 times (average 15 times). Patients who complained of severe spasms had spasms of longer duration and of more frequent occurrence. Just after waking in the morning and during Sunday night, spasms tended to occur more frequently. On the contrary, after exercise, spasms were less frequent and were less severe. There were 2 types of spasms in the trunk, tonic spasms and clonic spasms. Tonic spasms in the trunk were observed in 11 patients and clonic spasms in 5. Spasms in the trunk resulted in sensations of difficulty in breathing and nausea in some patients. There were 3 types of spasms in both upper and lower limbs, flexor spasms (tonic flexor spasms), extensor spasms (tonic extensor spasms), and clonic spasms (alternating flexor and extensor spasms). In the upper limbs, flexor spasms were seen in 4 patients, extensor spasms in 5, and clonic spasms in 5 . In the lower limbs; flexor spasms were seen in 7 patients, extensor spasms in 12, and clonic spasms in 9 . There was a wide range of variation in the pattern of spasms depending on which limb was involved and what types of spasms occurred. Even in an individual patient, the pattern of each spasm could be different. Based on the types of spasms in the limbs only, we could classify those spasms into 4 patterns. The pattern of flexor spasms in the upper limbs and extensor spasms in the lower limbs was found in 7 patients. The pattern of extensor spasms in both upper and lower limbs was found in 6 patients. The pattern of extensor spasms in the upper limbs and flexor spasms in the lower limbs was found in 6 patients. The pattern of flexor spasms in both upper and lower limbs was found in 4 patients.

Every spasm was always accompanied by a trigger. (Fig. 1). Active motion by patients themselves such as voluntary motion, yawn and cough, and psychological excitement acted as triggers for spasms. Passive motions by assistants also acted as triggers of spasms. Turning of the patient by nurses or nursing aides was found as a trigger of spasms in all patients. ROM exercise (passive movement for maintaining range of motion) by nurses or other staff acted frequently as trigger (Fig. 2). Other passive motions which triggered spasms were transfers, cleaning of the body, dressing, urination and evacuation of bowels, etc. These activities were also affected by the spasms.

Eleven of the 13 patients complained that interference with daily activities was caused by spasms. Activities carried out by patients, such as eating with 


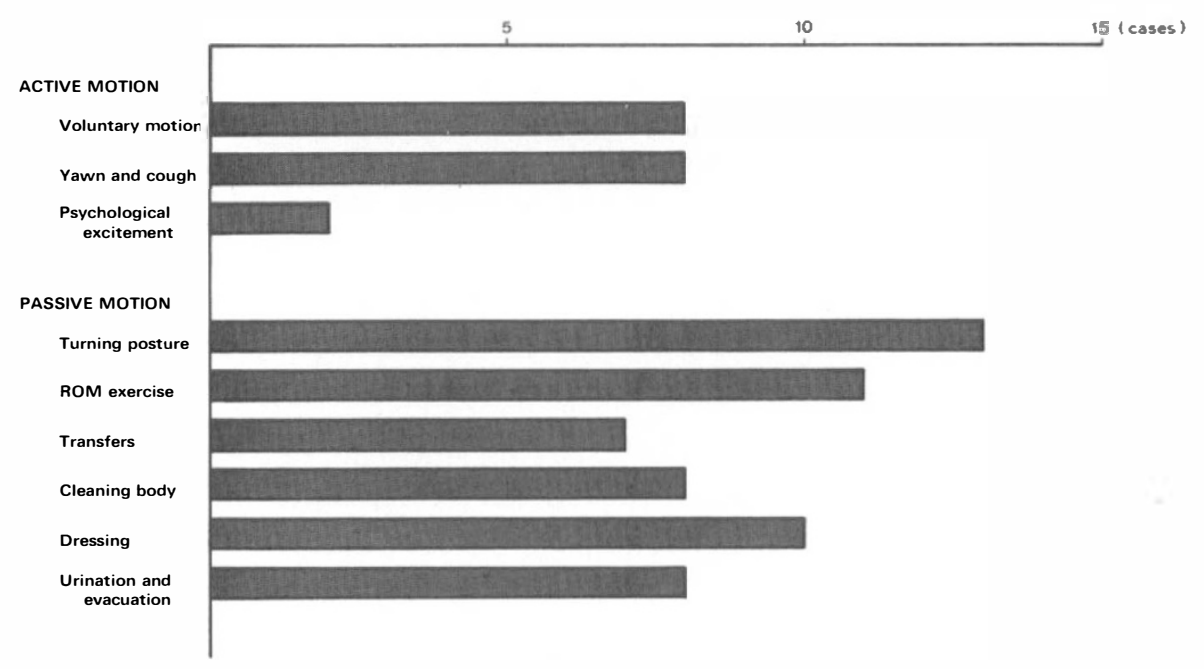

Figure 1 Triggers which elicited spasms.

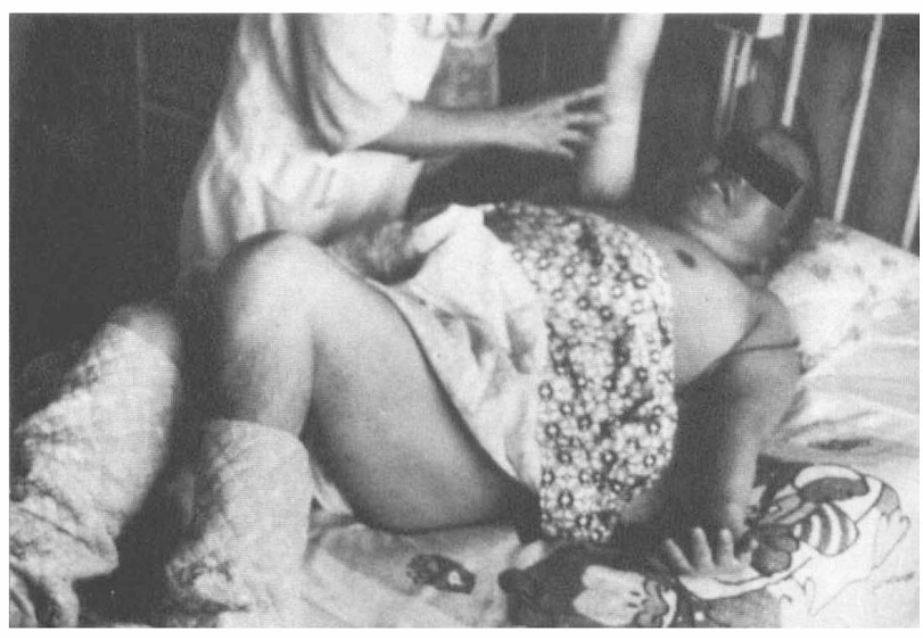

Figure 2 Extensor spasms of the left fingers elicited by passive motion of the right shoulder.

self-help devices and moving around in the wheelchair were interfered with by spasms. Most activities which were interfered with by spasms acted also as triggers for spasms. Spasms interfered with sleep after turning during the night in 7 patients (Fig. 3).

\section{Discussion}

It is a well-known fact that there are prolonged, uncontrolled excessive contractions of skeletal muscles occurring over the entire paralysed area in patients with spinal cord injuries. However, many authors have used various different terms to define these phenomena. Kuhn (1950) termed them flexor spasms, alternating flexor and extensor spasms, and predominant extensor spasms; Kaplan et al. (1982), used the term 'spasticity'; Dimitrijevic and Nathan (1967) 


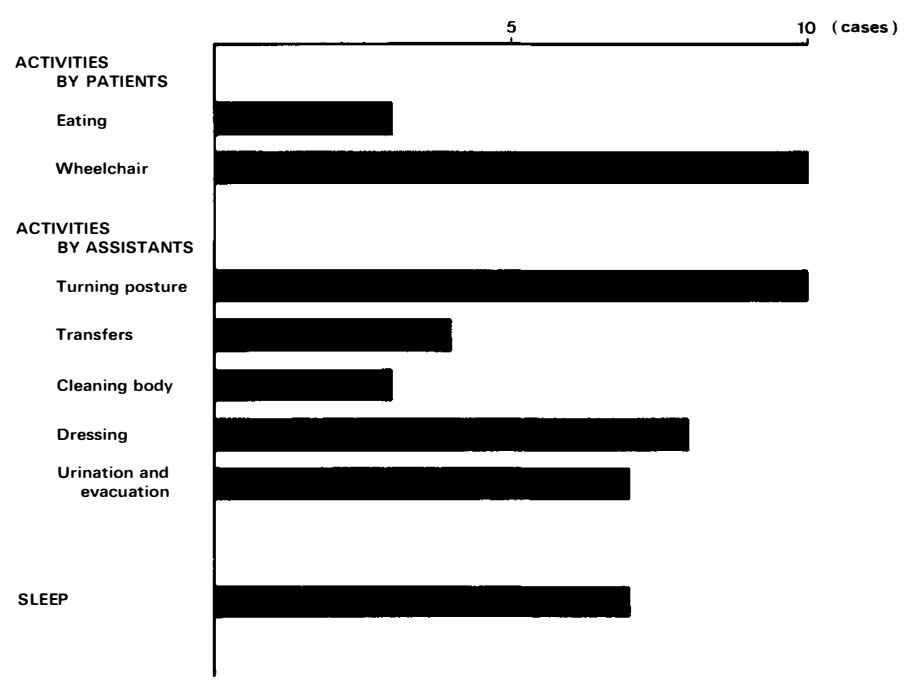

Figure 3 Activities of daily living which were interfered by spasms.

used the terms 'spasticity or involuntary spasms'; Guttmann (1973) used the term 'reflex automatism of the isolated cord'. Furthermore, there have been no suitable clinical tests to evaluate these phenomena in general (Merritt, 1981; Decker and Hall, 1986). Bajd and Vodovnik (1984) evaluated spasticity quantitatively by a pendulum test. However, according to our experience using the pendulum test to evaluate the effects of electrical stimulation on those patients (Kawamura et al., 1987), we found there were some patients who had severe and frequent spasms, but in whom both results of the pendulum test and of measuring resistance to passive motion were normal. Finally, we believe that we can evaluate those patients objectively and quantitatively only by clinical observation and by recording the EMG during 24 hours. Stenehjem et al. (1987) evaluated these patients by recording the EMG for time periods up to 24 hours using a Holter monitor. We are now also planning to measure EMG for 24 hours or more using the computerised recorder (Makikawa et al., 1987). This study was carried out as a part of our project concerning the development of quantitative measuring method of spasms in patients with spinal cord injuries.

Generally, it is said that the incidence of spasticity in patients with spinal cord injuries varies from 11 to $65 \%$ (Kaplan et al., 1962). In this study, all the patients were quadriplegic for 6 months or more and suffered from spasms. Tonic and clonic spasms were seen in their trunk, whereas flexor spasms, extensor spasms, and clonic spasms were seen in their limbs. Based on spasms in limbs, the pattern of spasms could be classified into four types. Generally, extensor spasms tended to occur more in the lower limbs than the upper limbs. The duration of spasms was a few seconds and the frequency was 10 times or more per day. The patients whose spasms were severe tended to be longer in duration and more frequent in occurrence. No spasm occured without trigger. In most patients the spasms interfered with the activities of daily living and with sleep. It is interesting to note that the many activities which were interfered with by spasms also acted as triggers of spasms. 


\section{Conclusion}

Spasms in patients with spinal cord injuries still continue to be a significant problem. Development of treatment and measuring methods of spasms is of great importance.

\section{Acknowledgement}

We thank M. Masatoshi, PT and S. Hayashi, PT for their co-operation for this study.

\section{References}

BAjd T, Vodovnik L 1984 Pendulum testing of spasticity. Fournal Biomedical Engineering 6:916.

DeCKer M, Hall A 1986 Physical therapy in spinal cord injury. In: Bloch RF, Basbaum M (eds) Management of Spinal Cord Injuries. Williams \& Wilkins, Baltimore, pp 320-347.

Dimitrijevic MR, NATHAN PW 1967 Studies of spasticity in man. 1. Some features of spasticity. Brain 90:1-30.

GutTMANN L 1973 Patterns of reflex disturbances. In: Spinal Cord Injuries. Blackwell, Oxford, pp 219-236.

Kaplan LI, Grynbaum BB, Lloyd KE, Rusk HA 1962 Pain and spasticity in patients with spinal cord dysfunction. Results of a follow-up study. FAMA 182:918-925.

KaWAMURA J, ISE M, MATSUYA M, et al. 1987 Clinical use of electrical stimulation for reducing spinal spasticity. Advances in External Control of Human Extremities IX, Bélgrade, pp 353362.

KUHN RA 1950 Functional capacity of the isolated human spinal cord. Brain 73:1-51.

Makikawa M, Horio H, Hasegawa T Development of a medical information collection robot and its clinical application. In preparation for publication.

MERRITT JL 1918 Management of spasticity in spinal cord injury. Mayo Clinical Proceedings 56:614-622.

STENEHJEM J, SwENSON JR, GRANGE TS 1987 Spasticity evaluation by ambulatory EMG. Advance in External Control of Human Extremities IX, Belgrade, pp 385-392. 